\title{
Radiotherapy for esophageal carcinoma: dose, response and survival
}

This article was published in the following Dove Press journal: Cancer Management and Research

\section{Yijun Luol,* \\ Qingfeng Mao ${ }^{2,3, *}$ \\ Xiaoli Wang' \\ Jinming $\mathrm{Yu}^{3}$ \\ Minghuan $\mathrm{Li}^{3}$ \\ 'Department of Oncology, The People's Hospital of Jiangxi, Nanchang, ${ }^{2}$ School of Medical and Life Sciences, University of Jinan-Shandong Academy of Medical Sciences, ${ }^{3}$ Department of Radiation Oncology and Radiology, Shandong Cancer Hospital Affiliated to Shandong University, Jinan, China}

*These authors contributed equally to this work
Correspondence: Minghuan Li Department of Radiation Oncology and Radiology, Shandong Cancer Hospital Affiliated to Shandong University, Jiyan Road 440, Jinan 250I I7, Shandong, China Tel +8653167626112

Fax +8653187984079

Email Sdlmh2014@163.com

\begin{abstract}
Esophageal cancer (EC) is an extremely aggressive, lethal malignancy that is increasing in incidence worldwide. At present, definitive chemoradiotherapy is accepted as the standard treatment for locally advanced EC. The EC guidelines recommend a radiation dose of $50.4 \mathrm{~Gy}$ for definitive treatment, yet the outcomes for patients who have received standard-dose radiotherapy remain unsatisfactory. However, some studies indicate that a higher radiation dose could improve local tumor control, and may also confer survival benefits. Some studies, however, suggest that high-dose radiotherapy does not bring survival benefit. The available data show that most failures occurred in the gross target volume (especially in the primary tumor) after definitive chemoradiation. Based on those studies, we hypothesize that at least for some patients, more intense local therapy may lead to better local control and survival. The aim of this review is to evaluate the radiation dose, fractionation strategies, and predictive factors of response to therapy in functional imaging for definitive chemoradiotherapy in esophageal carcinoma, with an emphasis on seeking the predictive model of response to CRT and trying to individualize the radiation dose for EC patients.
\end{abstract}

Keywords: esophageal cancer, radiation dose, altered fractionation, predictive factors, individualization

\section{Introduction}

Esophageal cancer (EC) is a common cause of cancer death around the world. ${ }^{1,2}$ In East Asia, squamous cell carcinoma (SCC) is the most common type of EC, whereas adenocarcinoma is predominant in Western countries. ${ }^{3,4}$ These two types may represent different diseases, each with a distinct pathogenesis, epidemiology, prognosis, and tumor biology, including the pattern of lymph node metastasis. ${ }^{5}$

At present, definitive concurrent chemoradiotherapy (CCRT) is the basic strategy for locally advanced inoperable EC patients based on the results of the pivotal study of Intergroup Radiation Therapy Oncology Group (RTOG)-8501, which significantly improved the local control (LC) and overall survival (OS) with CCRT compared with radiotherapy (RT) alone. ${ }^{6}$ Furthermore, another RTOG study (\#90-12) found that escalating the dose to 64.8 Gy did not confer a benefit compared with standard doses and may have contributed to a higher incidence of treatment-related death. ${ }^{7}$ In the landmark INT0123 trial, dose escalation from 50.4 to 64.8 Gy also did not increase OS; this may have been due to higher toxicities and no therapeutic gain in dose escalation for pathological complete responses (pCRs) after planned radiation. ${ }^{8}$ On the basis of these results, 50.4 Gy has become the accepted standard dose in Europe and North America 
for patients undergoing definitive CRT (DCRT). Although radiation dose escalation has failed to improve $\mathrm{LC}$ or OS, a dose of 60.0 Gy or more is more popular in Asian countries, where SCC is the predominant histological type. ${ }^{9,10}$ However, the prognosis for EC patients treated by standard-dose CRT was still disappointing, and $>50 \%$ of such patients eventually developed recurrence or distant metastases and died as a result. ${ }^{11}$ Many studies have been performed to describe patterns of recurrence after DCRT for EC, revealing that most failures in both early and advanced EC occurred in the gross target volume (GTV) (especially in the primary tumor) after CRT. ${ }^{12-18}$ Significantly, the recurrent site in the patient undergoing surgery occurred mainly in the locoregional node as well as distant metastasis, while in patients treated with DCRT it occurred mainly in the primary lesion. ${ }^{19-21}$ This indicates that the standard dose (50.4 Gy in 28 fractions), at least for some patients, may be inadequate to achieve a high probability of LC. Hence, for EC patients treated with DCRT, efforts should be made to intensify the treatment to improve the LC rate, which may lead to survival benefits.

In addition, on the basis of the theory of radiation biology, a radiation dose of 45-50 Gy is adequate to control microscopic tumors, 60 Gy or more is required to control gross tumors, and nearly $100 \mathrm{~Gy}$ is needed to cure solid tumors at conventional fractionation. ${ }^{22}$ In recent years, continuous advancements in RT technology over the past decades have allowed for EC now being able to be treated with 3-dimensional (3D) treatment planning, including intensity-modulated RT (IMRT), volumetric-modulated arc therapy (VMAT) and helical tomotherapy. These modern techniques allow the oncologist to deliver higher doses of radiation with more precision to the tumor and with less toxicity to the surrounding normal tissue, which has dramatically reduced morbidity. ${ }^{23,24}$ Thus, with the improvements in RT techniques and chemotherapy regimens, the question has been raised as to whether high-dose RT given concurrently with effective chemotherapy could achieve similar or better survival rates compared with the standard treatment, especially for esophageal SCC (ESCC).

The aim of this review is to evaluate the radiation dose, fractionation strategies, and predictive factors of response to therapy in functional imaging for definitive CRT in EC, with an emphasis on seeking the predictive model of response to CRT and trying to individualize the radiation dose for EC patients.

\section{Higher dose RT for DCRT}

Continuous advancements in RT technology allow the oncologist to deliver higher radiation doses to the tumor with less toxicity to the surrounding normal tissue. This has led to a dramatic decline in morbidity. ${ }^{23,24}$ Furthermore, the simultaneous integrated boost (SIB) technique has been applied to many tumors, including in EC. Use of an SIB technique enables delivery of a higher dose to the primary tumor (2.2 Gy/f), whereas lower doses to the subclinical disease $(1.8 \mathrm{~Gy} / \mathrm{f}){ }^{25,26}$

With the improvements in treatment techniques, some researchers have tried to address the potential benefit of a higher radiation dose for EC. In the retrospective analysis by Suh et al the results also showed that patients who received a total dose $\geq 50.4$ Gy of RT had significantly better loco-regional control (LRC, 69\% vs 32\%, $p<0.01$ ) and progression-free survival (PFS, $47 \%$ vs $20 \%, p=0.01$ ), than patients receiving $<50$ Gy when treated with concurrent chemotherapy. High-dose radiation $\geq 50.4$ Gy showed no significant OS benefit for patients with EC (28 vs 18 months, $p=0.26) .{ }^{27} \mathrm{He}$ et al used modern radiation delivery techniques to determine whether high radiation dose could confer benefits in terms of LC or OS. ${ }^{28}$ The results showed that high radiation dose provided a significant lower rate of local recurrence $(17.9 \%$ vs $34.3 \%, p=0.024)$ compared with patients receiving low radiation dose. Furthermore, patients receiving high radiation dose did have a marginally better 5 -year local-regional recurrence-free survival $(68.7 \%$ vs $55.9 \%, p=0.052$ ) than in the low-dose group. The 5-year OS rate showed no significant difference between the two groups $(p=0.617)$. Although IMRT reduced the overall incidence of treatment-related toxicity, the rates of grade 3 or greater skin reaction and esophageal strictures were higher in the higher dose group. Considering the potential for better tumor LC but more severe toxicity with the higher radiation dose, He et al recommends that individualized treatment strategies be designed for patients treated with DCRT. Indeed, these studies found that radiation doses over 50.4 Gy could improve LC but not OS for EC patients.

However, some studies have shown that high-dose RT may provide a survival benefit. The study of Zhang et al found that the median survival time was 9 months for the lower dose group ( $\leq 51 \mathrm{~Gy}$ ) and 14.5 months for the higher dose group ( $>51 \mathrm{~Gy}$ ) among 69 patients treated with CCRT $(p=0.054) .{ }^{29}$ In line with these findings, Kim et al evaluated the correlation between radiation dose and survival for EC patients treated with DCRT. The results showed that patients in the high-dose group ( $\geq 60 \mathrm{~Gy}$ ) had significantly better 2-year LRC (69.1\% vs 50.3\%, $p=0.002$ ), median PFS (16.7 vs 11.7 months, $p=0.029$ ), and median OS (35.1 vs 22.3 months, $p=0.043)$ than in the low-dose group (<60 Gy). ${ }^{30}$ Similar to 
these results, a retrospective study found that higher radiation dose may bring a survival benefit for EC patients. ${ }^{31}$ Wolf et al also concluded that the use of radiation doses exceeding 54 Gy was associated with better OS $(p=0.002)$. ${ }^{32}$ Semrau et al in a retrospective analysis, showed that patients receiving high radiation dose had a greater 2-year OS $(26.8 \%$, vs $7.5 \% ; p=0.0001)$, and PFS (17.4\% vs 5.0\%; $p=0.0001)$ than the low-dose group. ${ }^{33} \mathrm{~A}$ pooled analysis by Song et al investigated whether high-dose ( $>60$ Gy) RT in DCRT could confer survival benefits compared with the low-dose RT for patients with EC. ${ }^{34}$ Those results showed advantages in response rate, 5-year OS, locoregional recurrence, and distant failure compared with the standard RT arm. However, there is no evidence from prospective randomized trials to support that a higher radiation dose could bring survival benefit for EC patients.

In a recently published Phase I dose-escalation study, Yu et al suggest that it is feasible to deliver up to 70 Gy $(2.8$ Gy/F) to the GTV based on positron emission tomography/ computed tomography (PET/CT) in EC patients. ${ }^{35}$ Thus, the use of a novel dose-escalation technique may be more likely than high-dose (>60 Gy) RT to lead to better LC and OS. A Phase II study by Chen et al evaluated the efficacy of radiation dose escalation using SIB in 60 EC patients. ${ }^{36}$ RT consisted of $66 \mathrm{~Gy}$ at $2.2 \mathrm{~Gy}$ per fraction to the gross tumor and $54 \mathrm{~Gy}$ at $1.8 \mathrm{~Gy}$ per fraction to subclinical diseases simultaneously. The result showed that the 1-year LRC, distant metastasisfree survival (DMFS), disease-free survival (DFS), and OS rates were $87.6 \%, 78.6 \%, 86.0 \%, 80.5 \%$, respectively. And the 2-year LRC, DMFS, DFS, and OS rates were $75.6 \%$, $64.4 \%, 86.7 \%, 72.7 \%$, respectively. This indicates that SIB combined with concurrent chemotherapy is feasible, with tolerable acute toxicities in EC patients, and a trend of significant improvements in LRC and OS was shown.

Radiation dose escalation for DCRT studies are summarized in Table 1. In summary, a few studies have demonstrated that higher doses than standard RT can improve local tumor control and may lead to better survival for EC patients. Although no evidence from Phase III prospective randomized trials support the additional survival benefit of dose-escalated RT in the whole population, these results may suggest that a subgroup of patients may benefit from limited dose escalation. Considering the potential survival benefits but more severe toxicity in the higher radiation dose group, the optimal radiation dose should be managed on an individual basis. Nevertheless, Phase III trials comprising a standard-dose arm using modern RT technique are warranted in the right subgroup population.

\section{Dose fractionation strategies for DCRT}

The prognosis for EC patients treated with conventional RT alone remains discouraging despite the advances in radiotherapeutic techniques. The low survival rate and high incidence of locoregional treatment failure in EC have initiated a modification of conventionally fractionated RT. Recent studies are consistent in revealing that the accelerated reproliferation of carcinoma stem cells after RT is an important reason for the failure of RT. ${ }^{37,38}$ Therefore, it is speculated that the rates of LC and survival can be improved by controlling the accelerated reproliferation of carcinoma stem cells after RT. In this respect, it has been revealed that 3-4 weeks after RT, carcinoma stem cells started to have accelerated reproliferation, which provides the theoretical principle for accelerated hyperfraction RT later in the treatment process.

Table I Studies regarding high-dose RT and/or conventional-dose RT in esophageal cancer

\begin{tabular}{|c|c|c|c|c|c|c|c|}
\hline Authors & Radiation dosage & $\begin{array}{l}\text { Radiation } \\
\text { technique }\end{array}$ & $\begin{array}{l}\text { No. of } \\
\text { patients }\end{array}$ & LCR, \% & $p$-value & os & $p$-value \\
\hline \multirow[t]{2}{*}{ Minsky et al ${ }^{8}$} & $50.4 \mathrm{~Gy}$ & 2D-RT & 109 & 52 (LFR) & $>0.05$ & $40 \%$ (2 year) & $>0.05$ \\
\hline & $64.8 \mathrm{~Gy}$ & & 109 & 56 & & $31 \%$ & \\
\hline \multirow[t]{2}{*}{ Zhang et $\mathrm{al}^{29}$} & $<5$ I Gy & 2D-RT & 43 & 19 (3 year) & 0.011 & $3 \%$ (3 year) & 0.054 \\
\hline & $\geq 5 \mathrm{I} G y$ & & 26 & 36 & & $13 \%$ & \\
\hline \multirow[t]{2}{*}{ Suh et $\mathrm{al}^{27}$} & $<60 \mathrm{~Gy}$ & 2D-RT & 49 & 32 (2 year) & $<0.01$ & I8 months (MST) & 0.26 \\
\hline & $\geq 60 \mathrm{~Gy}$ & & 77 & 69 & & 28 months & \\
\hline \multirow[t]{2}{*}{ He et $\mathrm{a}^{28}$} & $\leq 50.4 \mathrm{~Gy}$ & 3D-RT & 137 & 34.3 (LFR) & 0.024 & $33.0 \%$ (5 year) & 0.617 \\
\hline & $>50.4 \mathrm{~Gy}$ & & 56 & 17.9 & & $41.7 \%$ & \\
\hline \multirow[t]{2}{*}{ Kim et $\mathrm{al}^{30}$} & $<60$ Gy & 3D-RT or & 120 & 37.3 (5 year) & 0.02 & 22.3 months (MST) & 0.043 \\
\hline & $\geq 60 \mathrm{~Gy}$ & IMRT & 116 & 59.7 & & 35.1 months & \\
\hline \multirow[t]{2}{*}{ Chen et $\mathrm{al}^{31}$} & $50-50.5 \mathrm{~Gy}$ & 3D-RT & 324 & NR & NR & $14 \%$ (5 year) & $<0.05$ \\
\hline & $\geq 60 \mathrm{~Gy}$ & & 324 & NR & & $22 \%$ & \\
\hline \multirow[t]{2}{*}{ Chen et $\mathrm{al}^{36}$} & GTV 66 Gy /30f & SIB-IMRT & 60 & $78.6 \%$ (2 year) & - & $72.7 \%$ (2 year) & - \\
\hline & CTV 54 Gy/30f & & & & & & \\
\hline
\end{tabular}

Abbreviations: CTV, clinical target volume; GTV, gross target volume; IMRT, intensity modulated radiotherapy; LCR, local control rate; LFR, local failure rate; MST, median survival time; NR, not reported; OS, overall survival; RT, radiotherapy; SIB, simultaneous integrated boost. 
Several studies have investigated whether it would be possible to achieve a better curative effect from RT by adopting a late-course accelerated hyperfraction (LCAF). A Japanese Phase II study of accelerated hyperfraction plus 5-fluorouracil/cisplatin chemotherapy showed a promising result. ${ }^{39}$ Similarly, Shi et al ${ }^{40}$ initiated a study of late-course accelerated hyperfractionated RT (LCAFRT) for ESCC treatment and it yielded encouraging results. They found that patients with the LCAFRT regime could achieve a better 5-year survival rate ( $34 \%$ vs $15 \%$ for patients with conventional fractionation) and LC rate ( $55 \%$ vs $21 \%$ for patients with conventional fractionation). A prospective study by Zhao et al also revealed that the LCAFRT regimen offers better LC and survival compared to standard chemotherapy plus RT, as in the RTOG 85-01 and 94-05 trials. ${ }^{41} \mathrm{~A}$ meta-analysis from China strengthened the evidence supporting the therapeutic benefits of LCAFRT compared with conventional fractionation for EC. ${ }^{42}$ In addition, a randomized controlled trial was recently undertaken to investigate whether LCAF 3D conformal RT could achieve better results than conventional fraction (CF) ${ }^{43}$ The resulting data showed that the 1-, 2- and 3-year survival rates were 79.2\%, $56.3 \%$, and $43.8 \%$, respectively, in the LCAF group; and, in the CF group, the 1-, 2- and 3-year survival rates were 74\%, 54\%, and $36 \%$, respectively $(p=0.476$ ). The 1 -, 2 - and 3-year LC rates were $81.3 \%, 62.5 \%$, and $50 \%$, respectively, in the LCAF group; in the CF group, the 1-, 2- and 3-year LC rates were $78 \%, 58 \%$, and $42 \%$, respectively $(p=0.454)$. In the CF group, the incidence of radiation-induced esophagitis was lower than that in the LCAF group ( $72 \%$ vs $93.8 \%$; $p=0.008$ ). It was concluded that EC patients in the LCAF group did have a slightly improved survival compared to those who received RT using conventional fractionation; the radiation toxicities, however, were greater in the LCAF group than those in the CF group. In a Phase III randomized study of LCAFRT plus concurrent chemotherapy for patients with ESCC, Zhao reported patients who received LCAFRT with concurrent chemotherapy had a tendency to better survival. But the incidence rates of grade 3 and 4 toxicities seemed higher in the LCAHRT + CT arm $(46 \%)$ than those in the LCAHRT arm $(25 \%)$, and the grade 5 toxicities for the two group were $6 \%$ and $0 \%$, respectively. ${ }^{44}$

These studies suggest that the accelerated hyperfractionated schedules were effective but with an increased incidence of acute III-IV grade toxicity, which limited the combination of concurrent chemotherapy with RT. The advent of modern RT techniques and low-toxicity chemotherapy drugs may improve the clinical efficacy. The medical effects and safety observations of this kind of combination should be verified in prospective trials.

\section{Future thinking}

Much evidence has shown that there is a positive correlation between OS rate and the scale of chemoradiation in the histopathological response of patients with EC. ${ }^{45-47}$ Patients with CRs had a 5-year OS rate of $61.6 \%$, which is higher than patients with an incomplete response or no response. ${ }^{45}$ We speculated that if more patients with incomplete or no responses after planned radiation could achieve CR through limited radiation dose escalation, their prognosis would be better. Besides, this scheme would avoid an increase in toxicity and a decrease in survival caused by dose escalation in patients with CRs.

But how to predict the response to therapy? A great number of studies have reported the response rates after CCRT in EC. ${ }^{6,8,16-18,48}$ The CR rate by stage was $89.7 \%-97 \%$ for T1, $50 \%-60 \%$ for T2 to T3, and $17 \%-39 \%$ for T4. For early-stage EC, most patients achieved a CR after CRT, while for locally advanced patients, CRT generally resulted in CR rates of $20 \%-50 \%$. Apart from the tumor stage, patients with EC receiving RT also showed disparate treatment responses. Unfortunately, a large number of patients were resistant to CRT, which resulted in persistent disease or immediate local failure. For radiosensitive patients, the standard radiation dose of 50.4 Gy may be sufficient to obtain a pCR. But for the resistant lesions or advanced-stage EC, it is difficult to achieve a pCR at this dose. Even a dose escalation could not produce a better response; it may merely increase treatmentrelated toxicities in such refractory cases. We speculated that a subgroup of patients with partial remission after the standard radiation of 50.4 Gy could achieve better responses through limited radiation dose escalations without increased treatment toxicities. Hence, the identification of the predictive and prognostic factors will help to guide the oncologist in making informed decisions regarding the optimal radiation dose for treating ESCC, and indicate who have greater possibility benefit from limited radiation dose escalation.

\section{Predictive value of fluorodeoxyglucose (FDG) PET/CT parameters}

The ability to identify some factors to predict or assess treatment response at an early stage after the start of treatment would be of great value. Extensive research shows that metabolic-related parameters, such as standardized uptake value (SUV), metabolic tumor volume (MTV), and total lesion glycolysis (TLG) of the primary tumor have the potential to become valuable predictors and prognostic biomarkers in EC patients. ${ }^{49-52}$ 
Kato et al assessed the potential value of metabolicrelated parameters in predicting the response to treatment. ${ }^{53}$ The $\mathrm{SUV}_{\text {mean }}$ before CRT in the non-CR group and the CR group were 10.2 and 4.9 , respectively. The $\mathrm{SUV}_{\text {mean }}$ after treatment in the two groups was 3.7 and 1.4, respectively. The changes in SUV of the CR patients were significantly lower than those of the non-CR patients $(p<0.05)$. The author concluded that the $\mathrm{SUV}_{\text {mean }}$ before CRT of the primary tumor has the potential to become a valuable predictor for response $(p<0.05)$. Similarly, Atsumi et al performed a study to assess the efficacy of metabolic-related parameters for the prediction of response in DCRT for EC. ${ }^{54}$ The results showed that the $\mathrm{SUV}_{\text {max }}$ values for the CR arm were higher than that in the non-CR arm, and all 18 patients in the low-SUV group had a CR. The data suggest that the SUV max $_{\text {mas a valuable }}$ predictor for response. However, Javeri et al evaluated the initial standardized unit value of FDGPET and its association with the degree of pathological response after CRT. ${ }^{55}$ Their work revealed that SUV higher than the median (10.1) was associated with a better pathological response $(p=0.06)$. Similarly, Levine et al and Rizk et al also reported a high

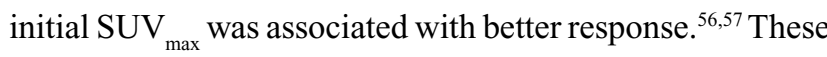
conflicting results could be potentially attributed to differences in patient populations, tumor histology types, grouping criteria, as well as treatment, but they might also indicate that predictive value of SUV values are unreliable.

Recently, some studies have considered that $\mathrm{SUV}_{\text {max }}$, which reflects only a single point in the tumor, may not always be representative of the whole tumor. ${ }^{58}$ In contrast to $\mathrm{SUV}_{\text {max }}$, volumetric parameters such as MTV and TLG represent the dual characteristics of tumor volume and the degree of tumor uptakes FDG. ${ }^{59}$ Therefore, volumetric parameters based on 18F-FDG PET/CT have been proposed as a more valuable biomarker for predicting survival or response to CRT than $\mathrm{SUV}_{\max }$ in patients with EC. Roedl et al evaluated the value of volumetric parameters in predicting response to CRT in patients with EC. They found that a decrease in the metabolic tumor diameter between pre- and posttreatment was the single best predictor of tumor response and survival outcome. ${ }^{60} \mathrm{In}$ another study, Jayachandran et al evaluated the value of MTV on PET scanning in predicting response to CRT in patients with EC. They found that the MTV2.5 and TLG2.5 were valuable markers for predicting the tumor response. ${ }^{61}$ Another study also showed that the changes in MTV and TLG between pre- and posttreatment were more precise for predicting pathological response than $\Delta \mathrm{SUV}_{\max }{ }^{62}$ However, they showed that none of these parameters were very accurate in predicting a pCR and that the volumetric parameters had a marginally higher accuracy than $\mathrm{SUV}_{\text {max }}$ in predicting treatment response to CRT. Overall, available data suggest that these metabolic parameters may be useful as predictors of treatment response, while the ability to predict accurately is still limited.

\section{Predictive value of $\mathrm{CT}$ perfusion parameters}

CT perfusion is a promising imaging tool in oncology; it can visualize changes in the tumor's vascular physiology and introduces elements of functional diagnostics in morphological imaging. ${ }^{63,64}$ Respecting this fact could be potentially useful in monitoring the response of the tumor to the CRT.

Stefanovic et al evaluated the value of the CT perfusion parameters in predicting response to $\mathrm{CRT} .{ }^{65} \mathrm{In}$ their study, 40 patients with SCC were reevaluated after CRT. The CT perfusion parameter values after the CRT were significantly correlated with tumor regression grade. These results showed that CT perfusion imaging can predict the response to CRT. Hansen et al also reported that $\mathrm{CT}$ perfusion parameters could be an early predictor of treatment response to CRT in EC. ${ }^{66}$

To further investigate the utility of each perfusion parameter for predicting histopathologic response in EC following chemoradiation, a great deal of research was performed. In the study performed by Hayano et al, they found that higher baseline blood flow (BF), higher baseline blood volume (BV) and low mean transit time (MTT) correlated significantly with a good response ${ }^{67}$ In another EC study, Makari et al examined changes in tumor perfusion before and after chemoradiation in ESCC. ${ }^{6}$ The results showed that responders had a significantly higher baseline BF and a significantly shorter baseline MTT than non-responders, while BV did not in ESCC. Similar to the findings already mentioned, Djuric-Stefanovic et al questioned whether the CT perfusion parameters could be useful to predict the pCR of EC to CRT. ${ }^{69}$ The results showed that $\mathrm{BF}_{\text {post-CRT }}$, $\mathrm{BV}_{\text {post-CRT }}$, and permeability surface post-CRT were significantly lower and $\mathrm{MTT}_{\text {post-CRT }}$ was significantly higher in the pCR group. The investigators concluded that CT perfusion parameters enable accurate prediction of $\mathrm{pCR}$ of EC to CRT, which could be useful in improving patient selection for further treatment.

In conclusion, both FDG PET parameters and CT perfusion parameters could be a good predictor for treatment response. Such predictive factors could help to identify the subgroups that are more likely to benefit from radiation dose escalation.

\section{Individualized radiation dose escalation based on decrease of tumor FDG uptake} The available data indicate that a decrease in tumor FDG uptake correlates with OS and pathological response for 
patients with EC. ${ }^{70}$ Cuenca et al performed a study showing that metabolic response during CRT for locally advanced EC has a great prognostic value. ${ }^{71}$ Using a $50 \%$ decrease in $\mathrm{SUV}_{\text {max }}$ as a cut-off, the 2-year OS in the good metabolic responders and poor responders was $62 \%$ and $27 \%$, respectively ( $p=0.016$ ). Similarly, Javeri et al declared that the higher the decrease of tumor FDG uptake after treatment, the better the survival of EC patients. ${ }^{72}$ Metabolic response using PET/ $\mathrm{CT}$ is a surrogate for histopathological response in predicting sensitivity to treatments of patients with EC. ${ }^{73}$ Evaluating the decrease in tumor FDG uptake could help to identify good responders to CRT. Thus, individualized radiation dose escalation based on decrease in tumor FDG uptake after standard radiation of 50.4 Gy could be feasible. According to the European Organization for Research and Treatment of Cancer criteria, metabolic response on FDGPET was divided into the following four types: complete metabolic response (CMR), partial metabolic response (PMR), stable metabolic disease, and progressive metabolic disease. ${ }^{74,75} \mathrm{CMR}$ was defined as complete resolution of $18 \mathrm{~F}-\mathrm{FDG}$ uptake within the tumor volume so that it was indistinguishable from surrounding normal tissue. On the basis of our conjecture, patients with CMR may not need to receive dose escalation after the standard radiation of 50.4 Gy, which may reduce the treatment-related toxicities. Patients with PMR may benefit from dose escalation after a conventional radiation dose of $50.4 \mathrm{~Gy}$. Of those with minor residual tumor after planned radiation, limited dose escalation would achieve CMR without increasing toxicities. These patients could benefit the most from dose escalation. For those with major residual tumor and no metabolic response after planned radiation, limited dose escalation may not change the persistence of local disease because of tumor resistance to CRT. Above all, it is necessary to determine the feasibility of individualized radiation dose escalation after planned chemoradiation based on the decrease in tumor FDG uptake.

\section{Individualized radiation dose escalation based on flow-metabolic phenotypes}

It has been reported that malignant tumors differ in terms of BF perfusion and glucose metabolism phenotype. ${ }^{76-78}$ For example, there are: 1) high-perfusion high-metabolism tumors; 2) low-perfusion low-metabolism tumors; 3) highperfusion low-metabolism tumors; and 4) low-perfusion high-metabolism tumors (Figure 1). A combined assessment of the flow-metabolic phenotype of EC using integrated 18F-FDG PET/perfusion CT may be of additional value in assessing the response to therapy as well as in identifying the patients who might be more likely to benefit from
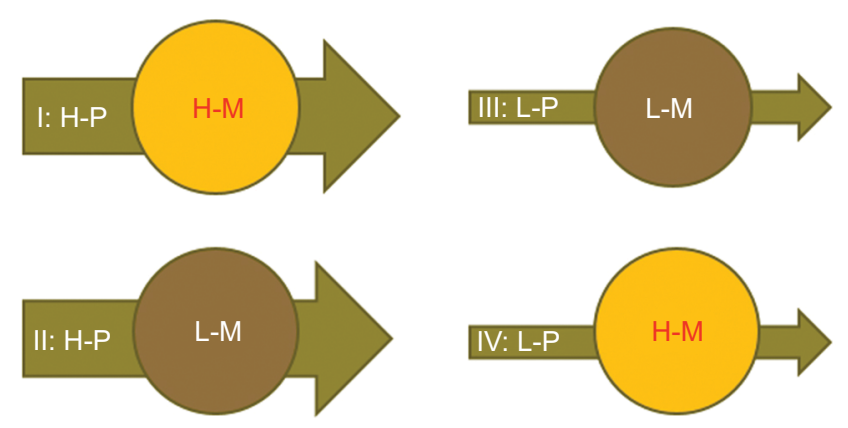

Figure I Representative different flow-metabolic phenotypes.

Notes: I: H-P and H-M; II: H-P and L-M; III: L-P and L-M; IV: L-P and H-M.

Abbreviations: H-P, high-perfusion; H-M, high-metabolism; L-P, low-perfusion; L-M, low metabolism.

radiation dose escalation. Several studies have investigated the relationship between $18 \mathrm{~F}-\mathrm{FDG}$ PET and perfusion CT, demonstrating that the balance between tumor vascularity and glucose metabolism offers massive information regarding tumor treatment response. ${ }^{76-78}$ Tumors with matched high perfusion and glucose metabolism show a constitutive up-regulation of angiogenesis and metabolism; tumors with matched low perfusion and glucose metabolism likely reflect necrosis; whereas tumors that present with low perfusion and high glucose metabolism show an adaptation to hypoxia. ${ }^{79}$ Thus, integrated 18F-FDG PET/perfusion CT makes it possible to distinguish different phenotypes; this may be useful in guiding individualized dose escalation based on functional imaging in patients with EC.

\section{Conclusion}

In conclusion, the available data reveal that LC after DCRT for EC remains a problem and that most local failures occur within the primary tumor. This indicates that the standard RT dose (50.4 Gy in 28 fractions) may be inadequate to achieve a high probability for LC for some subgroup patients. It is warranted to explore potential ways of improving LC, including IMRT and VMAT techniques, radiation dose escalation, and the use of more effective dose fractionation strategies. Some studies have found that the use of a higher dose and late course of accelerated hyperfraction radiation may lead to better LC and survival for EC patients undergoing CRT. Nevertheless, there is no evidence from Phase III randomized trials to support the additional benefit of dose-escalated RT. Although there is a potential for better tumor LC, there is also an associated higher incidence of toxicity. Therefore, the higher radiation dose should be used with caution on an individual basis in patients with EC.

It has been reported that EC probably has variable sensitivities to CRT. Therefore further studies will be required 
to: 1) identify the factors involved in EC sensitivity to radiation; 2) determine the causes of recurrence and non-control from molecular biology perspectives; and 3) individually determine the target region of RT, fractionated dose, and total dose to increase both LC and survival rates and decrease the rates of metastasis in patients with EC.

\section{Acknowledgment}

This study was funded by the Natural Science Foundation of China (NSFC 81672995) and The Key Research and Development Program of Shandong Province (2016GSF201133).

\section{Disclosure}

The authors report no conflicts of interest in this work.

\section{References}

1. Torre LA, Bray F, Siegel RL, Ferlay J, Lortet-Tieulent J, Jemal A. Global cancer statistics, 2012. CA Cancer J Clin. 2015;65(2):87-108.

2. Kumagai K, Rouvelas I, Tsai JA, et al. Meta-analysis of postoperative morbidity and perioperative mortality in patients receiving neoadjuvant chemotherapy or chemoradiotherapy for resectable oesophageal and gastro-oesophageal junctional cancers. Br J Surg. 2014;101(4):321-338.

3. Wheeler JB, Reed CE. Epidemiology of esophageal cancer. Surg Clin North Am. 2012;92(5):1077-1087.

4. Ajani JA. Gastroesophageal cancers: progress and problems. J Natl Compr Canc Netw. 2008;6(9):813-814.

5. Song Y, Li L, Ou Y, et al. Identification of genomic alterations in oesophageal squamous cell cancer. Nature. 2014;509(7498):91-95.

6. Cooper JS, Guo MD, Herskovic A, et al. Chemoradiotherapy of locally advanced esophageal cancer: long-term follow-up of a prospective randomized trial (RTOG 85-01). Radiation Therapy Oncology Group. JAMA. 1999;281(17):1623-1627.

7. Minsky BD, Neuberg D, Kelsen DP, et al. Final report of intergroup trial 0122 (ECOG PE-289, RTOG 90-12): Phase II trial of neoadjuvant chemotherapy plus concurrent chemotherapy and high-dose radiation for squamous cell carcinoma of the esophagus. Int J Radiat Oncol Biol Phys. 1999;43(3):517-523.

8. Minsky BD, Pajak TF, Ginsberg RJ, et al. INT 0123 (Radiation Therapy Oncology Group 94-05) phase III trial of combined-modality therapy for esophageal cancer: high-dose versus standard-dose radiation therapy. J Clin Oncol. 2002;20(5):1167-1174.

9. Higuchi K, Koizumi W, Tanabe S, et al. Current management of esophageal squamous-cell carcinoma in Japan and other countries. Gastrointest Cancer Res. 2009;3(4):153-161.

10. Meng X, Wang J, Sun X, et al. Cetuximab in combination with chemoradiotherapy in Chinese patients with non-resectable, locally advanced esophageal squamous cell carcinoma: a prospective, multicenter phase II trail. Radiother Oncol. 2013;109(2):275-280.

11. Li M, Zhang X, Zhao F, Luo Y, Kong L, Yu J. Involved-field radiotherapy for esophageal squamous cell carcinoma: theory and practice. Radiat Oncol. 2016;11:18.

12. Bedenne L, Michel P, Bouché O, et al. Chemoradiation followed by surgery compared with chemoradiation alone in squamous cancer of the esophagus: FFCD 9102. J Clin Oncol. 2007;25(10):1160-1168.

13. Rawat S, Kumar G, Kakria A, Sharma MK, Chauhan D. Chemoradiotherapy in the management of locally advanced squamous cell carcinoma esophagus: is surgical resection required. $J$ Gastrointest Cancer. 2013;44(3):277-284.

14. Pöttgen C, Stuschke M. Radiotherapy versus surgery within multimodality protocols for esophageal cancer a meta-analysis of the randomized trials. Cancer Treat Rev. 2012;38(6):599-604.
15. Kawaguchi Y, Nishiyama K, Miyagi K, Suzuki O, Ito Y, Nakamura $\mathrm{S}$. Patterns of failure associated with involved field radiotherapy in patients with clinical stage I thoracic esophageal cancer. Jpn J Clin Oncol. 2011;41(8):1007-1012.

16. van Hagen P, Hulshof MC, van Lanschot JJ, et al. Preoperative chemoradiotherapy for esophageal or junctional cancer. $N$ Engl $J$ Med. 2012;366(22):2074-2084.

17. Kato K, Nakajima TE, Ito Y, et al. Phase II study of concurrent chemoradiotherapy at the dose of $50.4 \mathrm{~Gy}$ with elective nodal irradiation for Stage II-III esophageal carcinoma. Jpn J Clin Oncol. 2013;43(6): 608-615.

18. Rohatgi PR, Swisher SG, Correa AM, et al. Failure patterns correlate with the proportion of residual carcinoma after preoperative chemoradiotherapy for carcinoma of the esophagus. Cancer. 2005;104(7):1349-1355.

19. Nakagawa S, Kanda T, Kosugi S, Ohashi M, Suzuki T, Hatakeyama $\mathrm{K}$. Recurrence pattern of squamous cell carcinoma of the thoracic esophagus after extended radical esophagectomy with three-field lymphadenectomy. J Am Coll Surg. 2004;198(2):205-211.

20. Reid TD, Davies IL, Mason J, Roberts SA, Crosby TD, Lewis WG. Stage for stage comparison of recurrence patterns after definitive chemoradiotherapy or surgery for oesophageal carcinoma. Clin Oncol (R Coll Radiol). 2012;24(9):617-624.

21. Sugiyama M, Morita M, Yoshida R, et al. Patterns and time of recurrence after complete resection of esophageal cancer. Surg Today. 2012;42(8):752-758.

22. Fletcher GH. Clinical dose-response curves of human malignant epithelial tumours. Br J Radiol. 1973;46(541):1-12.

23. Li JC, Liu D, Chen MQ, et al. Different radiation treatment in esophageal carcinoma: a clinical comparative study. J BUON. 2012;17(3):512-516.

24. Chen YJ, Liu A, Han C, et al. Helical tomotherapy for radiotherapy in esophageal cancer: a preferred plan with better conformal target coverage and more homogeneous dose distribution. Med Dosim. 2007;32(3):166-171.

25. Leclerc M, Maingon P, Hamoir M, et al. A dose escalation study with intensity modulated radiation therapy (IMRT) in T2N0, T2N1, T3N0 squamous cell carcinomas (SCC) of the oropharynx, larynx and hypopharynx using a simultaneous integrated boost (SIB) approach. Radiother Oncol. 2013;106(3):333-340.

26. Yu WW, Zhu ZF, Fu XL, et al. Simultaneous integrated boost intensitymodulated radiotherapy in esophageal carcinoma: early results of a phase II study. Strahlenther Onkol. 2014;190(11):979-986.

27. Suh YG, Lee IJ, Koom WS, et al. High-dose versus standard-dose radiotherapy with concurrent chemotherapy in stages II-III esophageal cancer. Jpn J Clin Oncol. 2014;44(6):534-540.

28. He L, Allen PK, Potter A, et al. Re-evaluating the optimal radiation dose for definitive chemoradiotherapy for esophageal squamous cell carcinoma. J Thorac Oncol. 2014;9(9):1398-1405.

29. Zhang Z, Liao Z, Jin J, et al. Dose-response relationship in locoregional control for patients with stage II-III esophageal cancer treated with concurrent chemotherapy and radiotherapy. Int J Radiat Oncol Biol Phys. 2005;61(3):656-664.

30. Kim HJ, Suh YG, Lee YC, et al. Dose-response relationship between radiation dose and loco-regional control in patients with stage II-III esophageal cancer treated with definitive chemoradiotherapy. Cancer Res Treat. 2017;49(3):669-677.

31. Chen CY, Li CC, Chien CR. Does higher radiation dose lead to better outcome for non-operated localized esophageal squamous cell carcinoma patients who received concurrent chemoradiotherapy? A population based propensity-score matched analysis. Radiother Oncol. 2016;120(1):136-139.

32. Wolf MC, Zehentmayr F, Schmidt M, Hölzel D, Belka C. Treatment strategies for oesophageal cancer - time-trends and long term outcome data from a large tertiary referral centre. Radiat Oncol. 2012;7:60.

33. Semrau R, Herzog SL, Vallböhmer D, Kocher M, Hölscher AH, Müller RP. Prognostic factors in definitive radiochemotherapy of advanced inoperable esophageal cancer. Dis Esophagus. 2012;25(6):545-554. 
34. Song T, Liang X, Fang M, Wu S. High-dose versus conventional-dose irradiation in cisplatin-based definitive concurrent chemoradiotherapy for esophageal cancer: a systematic review and pooled analysis. Expert Rev Anticancer Ther. 2015;15(10):1157-1169.

35. Yu W, Cai XW, Liu Q, et al. Safety of dose escalation by simultaneous integrated boosting radiation dose within the primary tumor guided by (18)FDG-PET/CT for esophageal cancer. Radiother Oncol. 2015;114(2):195-200.

36. Chen J, Guo H, Zhai T, et al. Radiation dose escalation by simultaneous modulated accelerated radiotherapy combined with chemotherapy for esophageal cancer: a phase II study. Oncotarget. 2016;7(16): 22711-22719.

37. Withers HR, Taylor JM, Maciejewski B. The hazard of accelerated tumor clonogen repopulation during radiotherapy. Acta Oncol. 1988;27(2):131-146.

38. Trott KR. Cell repopulation and overall treatment time. Int J Radiat Oncol Biol Phys. 1990;19(4):1071-1075.

39. Jeremic B, Shibamoto Y, Acimovic L, et al. Accelerated hyperfractionated radiation therapy and concurrent 5 -fluorouracil/cisplatin chemotherapy for locoregional squamous cell carcinoma of the thoracic esophagus: a phase II study. Int J Radiat Oncol Biol Phys. 1998;40(5):1061-1066.

40. Shi XH, Yao W, Liu T. Late course accelerated fractionation in radiotherapy of esophageal carcinoma. Radiother Oncol. 1999;51(1):21-26.

41. Zhao KL, Shi XH, Jiang GL, Wang Y. Late-course accelerated hyperfractionated radiotherapy for localized esophageal carcinoma. Int $J$ Radiat Oncol Biol Phys. 2004;60(1):123-129.

42. Zhang YW, Chen L, Bai Y, Zheng X. Long-term outcomes of late course accelerated hyper-fractionated radiotherapy for localized esophageal carcinoma in Mainland China: a meta-analysis. Dis Esophagus. 2011;24(7):495-501.

43. Wang JH, Lu XJ, Zhou J, Wang F. A randomized controlled trial of conventional fraction and late course accelerated hyperfraction three-dimensional conformal radiotherapy for esophageal cancer. Cell Biochem Biophys. 2012;62(1):107-112.

44. Zhao KL, Shi XH, Jiang GL, et al. Late course accelerated hyperfractionated radiotherapy plus concurrent chemotherapy for squamous cell carcinoma of the esophagus: a phase III randomized study. Int J Radiat Oncol Biol Phys. 2005;62(4):1014-1020.

45. Tong DK, Law S, Kwong DL, Chan KW, Lam AK, Wong KH. Histological regression of squamous esophageal carcinoma assessed by percentage of residual viable cells after neoadjuvant chemoradiation is an important prognostic factor. Ann Surg Oncol. 2010;17(8):2184-2192.

46. Chao YK, Chan SC, Liu YH, et al. Pretreatment T3-4 stage is an adverse prognostic factor in patients with esophageal squamous cell carcinoma who achieve pathological complete response following preoperative chemoradiotherapy. Ann Surg. 2009;249(3):392-396.

47. Hammoud ZT, Kesler KA, Ferguson MK, et al. Survival outcomes of resected patients who demonstrate a pathologic complete response after neoadjuvant chemoradiation therapy for locally advanced esophageal cancer. Dis Esophagus. 2006;19(2):69-72.

48. Nishimura Y, Suzuki M, Nakamatsu K, Kanamori S, Yagyu Y, Shigeoka $\mathrm{H}$. Prospective trial of concurrent chemoradiotherapy with protracted infusion of 5-fluorouracil and cisplatin for T4 esophageal cancer with or without fistula. Int J Radiat Oncol Biol Phys. 2002;53(1):134-139.

49. Hatt M, Le Pogam A, Visvikis D, Pradier O, Cheze Le Rest C. Impact of partial-volume effect correction on the predictive and prognostic value of baseline 18F-FDG PET images in esophageal cancer. $J$ Nucl Med. 2012;53(1):12-20.

50. Muijs CT, Beukema JC, Pruim J, et al. A systematic review on the role of FDG-PET/CT in tumour delineation and radiotherapy planning in patients with esophageal cancer. Radiother Oncol. 2010;97(2):165-171.

51. Huh JW, Min JJ, Lee JH, Kim HR, Kim YJ. The predictive role of sequential FDG-PET/CT in response of locally advanced rectal cancer to neoadjuvant chemoradiation. Am J Clin Oncol. 2012;35(4):340-344.
52. Capirci C, Rubello D, Pasini F, et al. The role of dual-time combined 18-fluorodeoxyglucose positron emission tomography and computed tomography in the staging and restaging workup of locally advanced rectal cancer, treated with preoperative chemoradiation therapy and radical surgery. Int J Radiat Oncol Biol Phys. 2009;74(5):1461-1469.

53. Kato H, Fukuchi M, Miyazaki T, et al. Prediction of response to definitive chemoradiotherapy in esophageal cancer using positron emission tomography. Anticancer Res. 2007;27(4C):2627-2633.

54. Atsumi K, Nakamura K, Abe K, et al. Prediction of outcome with FDGPET in definitive chemoradiotherapy for esophageal cancer. $J$ Radiat Res. 2013;54(5):890-898.

55. Javeri H, Xiao L, Rohren E, et al. Influence of the baseline 18F-fluoro2-deoxy-D-glucose positron emission tomography results on survival and pathologic response in patients with gastroesophageal cancer undergoing chemoradiation. Cancer. 2009;115(3):624-630.

56. Levine EA, Farmer MR, Clark P, et al. Predictive value of 18-fluorodeoxy-glucose-positron emission tomography (18F-FDG-PET) in the identification of responders to chemoradiation therapy for the treatment of locally advanced esophageal cancer. Ann Surg. 2006;243(4):472-478.

57. Rizk NP, Tang L, Adusumilli PS, et al. Predictive value of initial PETSUVmax in patients with locally advanced esophageal and gastroesophageal junction adenocarcinoma. J Thorac Oncol. 2009;4(7):875-879.

58. Larson SM, Erdi Y, Akhurst T, et al. Tumor treatment response based on visual and quantitative changes in global tumor glycolysis using PET-FDG imaging. The visual response score and the change in total lesion glycolysis. Clin Positron Imaging. 1999;2(3):159-171.

59. Chung MK, Jeong HS, Park SG, et al. Metabolic tumor volume of [18F]-fluorodeoxyglucose positron emission tomography/computed tomography predicts short-term outcome to radiotherapy with or without chemotherapy in pharyngeal cancer. Clin Cancer Res. 2009;15(18):5861-5868.

60. Roedl JB, Colen RR, Holalkere NS, Fischman AJ, Choi NC, Blake MA. Adenocarcinomas of the esophagus: response to chemoradiotherapy is associated with decrease of metabolic tumor volume as measured on PET-CT. Comparison to histopathologic and clinical response evaluation. Radiother Oncol. 2008;89(3):278-286.

61. Jayachandran P, Pai RK, Quon A, et al. Postchemoradiotherapy positron emission tomography predicts pathologic response and survival in patients with esophageal cancer. Int J Radiat Oncol Biol Phys. 2012;84(2):471-477.

62. Stiekema J, Vermeulen D, Vegt E, et al. Detecting interval metastases and response assessment using 18F-FDG PET/CT after neoadjuvant chemoradiotherapy for esophageal cancer. Clin Nucl Med. 2014;39(10):862-867.

63. Miles KA, Lee TY, Goh V, et al; Experimental Cancer Medicine Centre Imaging Network Group. Current status and guidelines for the assessment of tumour vascular support with dynamic contrast-enhanced computed tomography. Eur Radiol. 2012;22(7):1430-1441.

64. Bellomi M, Viotti S, Preda L, D'Andrea G, Bonello L, Petralia G. Perfusion CT in solid body-tumours. Part II: clinical applications and future development. Radiol Med. 2010;115(6):858-874. Italian.

65. Djuric-Stefanovic A, Micev M, Stojanovic-Rundic S, Pesko P, Saranovic Dj. Absolute CT perfusion parameter values after the neoadjuvant chemoradiotherapy of the squamous cell esophageal carcinoma correlate with the histopathologic tumor regression grade. Eur J Radiol. 2015;84(12):2477-2484.

66. Lundsgaard Hansen M, Fallentin E, Lauridsen C, et al. Computed tomography (CT) perfusion as an early predictive marker for treatment response to neoadjuvant chemotherapy in gastroesophageal junction cancer and gastric cancer a prospective study. PLoS One. 2014;9(5):e97605.

67. Hayano K, Okazumi S, Shuto K, et al. Perfusion CT can predict the response to chemoradiation therapy and survival in esophageal squamous cell carcinoma: initial clinical results. Oncol Rep. 2007;18(4): 901-908. 
68. Makari Y, Yasuda T, Doki Y, et al. Correlation between tumor blood flow assessed by perfusion CT and effect of neoadjuvant therapy in advanced esophageal cancers. J Surg Oncol. 2007;96(3):220-229.

69. Djuric-Stefanovic A, Saranovic D, Micev M, et al. Does the computed tomography perfusion imaging improve the diagnostic accuracy in the response evaluation of esophageal carcinoma to the neoadjuvant chemoradiotherapy? Preliminary study. J BUON. 2014;19(1):237-244.

70. Onal C, Torun N, Guler OC, Yildirim BA. Prognostic value of metabolic response measured by $18 \mathrm{~F}$-FDG-PET in oesophageal cancer patients treated with definitive chemoradiotherapy. Nucl Med Commun. 2016;37(12):1282-1289.

71. Cuenca X, Hennequin C, Hindié E, et al. Evaluation of early response to concomitant chemoradiotherapy by interim 18F-FDG PET/CT imaging in patients with locally advanced oesophageal carcinomas. Eur J Nucl Med Mol Imaging. 2013;40(4):477-485.

72. Javeri H, Xiao L, Rohren E, et al. The higher the decrease in the standardized uptake value of positron emission tomography after chemoradiation, the better the survival of patients with gastroesophageal adenocarcinoma. Cancer. 2009;115(22):5184-5192.

73. Stiles BM, Salzler G, Jorgensen A, et al. Complete metabolic response is not uniformly predictive of complete pathologic response after induction therapy for esophageal cancer. Ann Thorac Surg. 2013;96(5):1820-1825.
74. Young H, Baum R, Cremerius U, et al. Measurement of clinical and subclinical tumour response using [18F]-fluorodeoxyglucose and positron emission tomography: review and 1999 EORTC recommendations. European Organization for Research and Treatment of Cancer (EORTC) PET Study Group. Eur J Cancer. 1999;35(13):1773-1782.

75. Kim MK, Ryu JS, Kim SB, et al. Value of complete metabolic response by (18)F-fluorodeoxyglucose-positron emission tomography in oesophageal cancer for prediction of pathologic response and survival after preoperative chemoradiotherapy. Eur J Cancer. 2007;43(9): 1385-1391.

76. Veit-Haibach P, Schmid D, Strobel K, et al. Combined PET/CT-perfusion in patients with head and neck cancers. Eur Radiol. 2013;23(1):163-173.

77. Goh V, Rodriguez-Justo M, Engledow A, et al. Assessment of the metabolic flow phenotype of primary colorectal cancer: correlations with microvessel density are influenced by the histological scoring method. Eur Radiol. 2012;22(8):1687-1692.

78. Goh V, Engledow A, Rodriguez-Justo M, et al. The flow-metabolic phenotype of primary colorectal cancer: assessment by integrated $18 \mathrm{~F}$ FDG PET/perfusion CT with histopathologic correlation. J Nucl Med. 2012;53(5):687-692.

79. Padhani AR, Miles KA. Multiparametric imaging of tumor response to therapy. Radiology. 2010;256(2):348-364.
Cancer Management and Research

\section{Publish your work in this journal}

Cancer Management and Research is an international, peer-reviewed open access journal focusing on cancer research and the optimal use of preventative and integrated treatment interventions to achieve improved outcomes, enhanced survival and quality of life for the cancer patient. The manuscript management system is completely online and includes

\section{Dovepress}

a very quick and fair peer-review system, which is all easy to use. Visit http://www.dovepress.com/testimonials.php to read real quotes from published authors. 